\title{
Human Vision Based Detection of Non-uniform Brightness on LCD Panels
}

\author{
Jee Hong Kim* and Brian A. Barsky** \\ *Division of Image Information Engineering, Pukyong National University, Busan 608-739, Korea \\ **Computer Science Division and School of Optometry, University of California, Berkeley CA 94720, U.S.A
}

\begin{abstract}
We propose a method to detect defects due to spatially non-uniform brightness on LCD panels by using a machine vision technique. The detection method is based on human vision so that proper subjective assessment experiments were conducted to investigate the correlation between the parameters related to non-uniformity and the degree how easily observable it is. The visibility of the defects reveals to depend mainly on the spatial gradient of brightness variation. Thus, in the proposed method, the spatial gradient that is calculated by using extracted contours will be utilized to detect the defects due to non-uniform brightness. The detection method comprises four parts: contour extraction, spatial gradient calculation, decision of defects, and display of defects. We applied the method to the images captured from practical LCD panels with non-uniformity defects and the results were consistent with detection by a human inspector.
\end{abstract}

\section{Introduction}

Progress in display technology brings products to market with a wide range of quality and price. Most display devices must guarantee high quality images without defects and thus any defects or resulting degradation in displayed image quality should be detected in production lines. As a typical flat panel display, the LCD (Liquid Crystal Display) is the dominant display device in the current market. Major electronics manufacturers produce LCD panels in huge volume; thus, productivity is an important issue and there is a need for an automated and effective method for defect inspection using machine vision.

There are several types of defects in LCD panels. Most defects are of a dot or spot shape $^{[1][2]}$ and a number of detection methods have been proposed for this type of defect.
However, there are some other defects which exhibit different properties from the defects that are of the dot and spot shapes. For example, defects due to spatial variation in brightness have the characteristics of very low spatial frequency. ${ }^{[3][4]}$ We refer to these types of defects as non-uniformity defects. Since conventional image processing techniques tend not to work for non-uniformity defects, ${ }^{[5]}$ there is a need for new effective detection methods.

In LCD panels, non-uniform luminance from backlight or some problems in fabrication process may cause such non-uniformity; this may be exhibited as blobs or stains with a variety of shapes and sizes. It is noteworthy that not all non-uniformity causes image degradation or defects. Sometimes it is apparent and other times it is not, depending on the extent to which the human visual system is sensitive to the nonuniformity. When the non-uniformity in

Machine Vision Applications in Industrial Inspection XIV, edited by Fabrice Meriaudeau, Kurt S. Niel, Proc. of SPIE-IS\&T Electronic Imaging, SPIE Vol. 6070, 60700P, @ 2006 SPIE-IS\&T · 0277-786X/06/\$15 
brightness is hard to discriminate visually, it can be neglected. ${ }^{[6][7]}$

The objective of the proposed detection method is to detect those defects due to spatially non-uniform brightness that would be apparent based on the human visual system. The intent is to develop a method that could be applied effectively to automated inspection systems using machine vision.

\section{Subjective Assessment on Visibility}

To investigate the correlation between the visibility of non-uniformity defects and physical parameters related to non-uniform brightness in an image, let us start by taking an example using the image captured from the real LCD panel with non-uniformity defects as shown in Fig. 1. In the image, we can observe some nonuniformity in spatial brightness over the whole region; how easily the non-uniform brightness can be observed depends on the spatial distribution of brightness.

Here, we consider two parameters related to spatial distribution, as follows: The deviation refers to the magnitude of the difference in brightness, that is, how much the brightness in a location differs from another location. The gradient refers to the spatial derivative of the brightness, that is, how rapidly the brightness varies.

In general, the larger the deviation is, the easier it is to observe the non-uniform brightness. But, it was also observed that the non-uniformity with less deviation was more observable than larger deviation when it had much larger gradient. Thus, there is a need for proper experiments to determine which parameter has more influence on visibility among the deviation and spatial gradient in brightness, and as well as to determine the amount of the deviation and gradient for just noticeable non-uniformity defects by human vision.

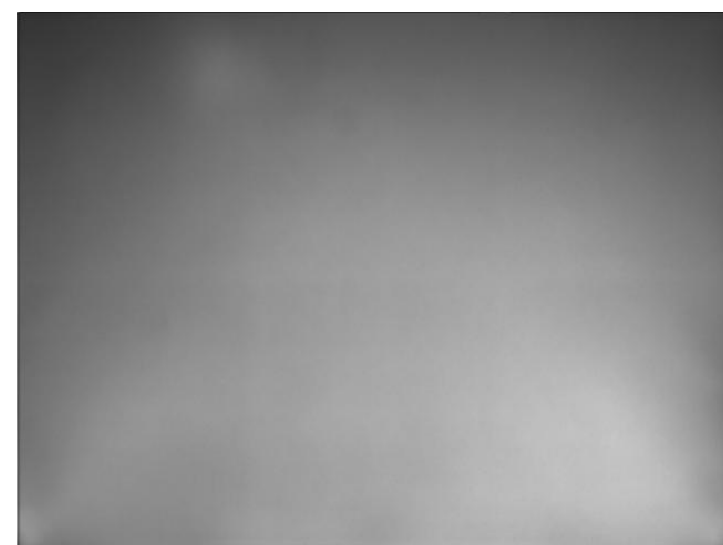

Fig. 1) Captured image from the LCD panels with non-uniformity defects

We conducted subjective experiments, using many rendered images with several values of deviation and gradient of brightness. There are several terms that we can use to describe brightness, such as luminance or associated brightness as the amount of sensation in human vision. ${ }^{[8]}$ or pixel values in images, and we assume that pixel values in captured images of LCD panels are approximately proportional to brightness, so the terms brightness and pixel values will be used synonymously.

In the experiment, we used the images containing the round shaped non-uniformity with a variety of deviation and spatial gradient. A normal distribution function was used to render non-uniformity with two-dimensional variation of brightness in the image, and the distribution of pixel values for the round shaped non-uniformity can be obtained by Eqn. 1). ${ }^{[9]}$

$$
\begin{array}{r}
f(x, y)=L+\frac{\Delta L}{\sigma \sqrt{2 \pi}} \exp \left(-\frac{\left(x-m_{x}\right)^{2}+\left(y-m_{y}\right)^{2}}{2 \sigma^{2}}\right) \\
\ldots . \text { Eqn. } 1)
\end{array}
$$

In Eqn. 1), $L$ is the pixel value for nominal brightness and $L$ is the amount of deviation; thus, pixel values vary from $\Delta L$ to $L-\Delta L$. The coordinates $\left(m_{x}, m_{y}\right)$ are the center of round shaped non-uniform brightness distribution and $\sigma$ means the standard deviation. Fig. 2) shows one of sample images with round shaped nonuniformity by Eqn. 1), where the brightness 
varies from $L$ to $L-\Delta L$ along the direction to the center.

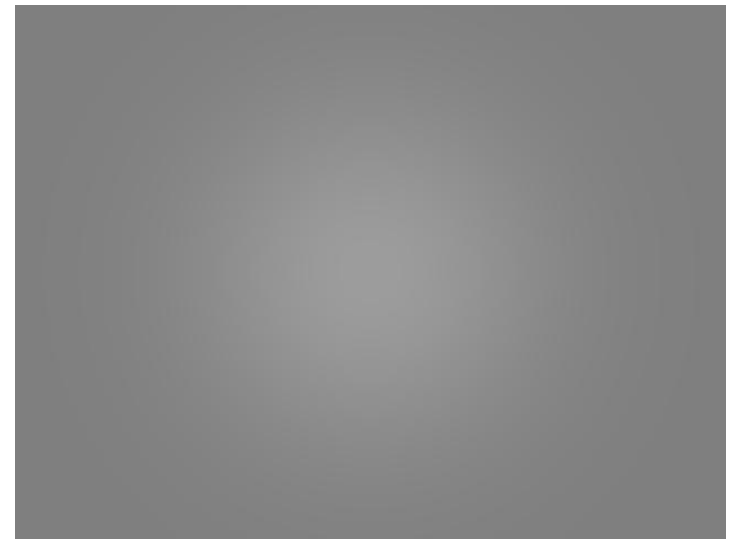

Fig. 2) Rendered images with round shaped nonuniformity

The subjective experiments were conducted using a display device which was a 15" LCD monitor with resolution $1024 \times 768$ and with nominal luminance of $150 \mathrm{~cd} / \mathrm{m} 2$ for a pixel value of 128 . Of course, the monitor used in the experiment had only slight non-uniformity.

The subjects understood the terms "nonuniformity", "degradation", and "defects". After viewing for two seconds, each subject was asked: "Did you perceive the defects due to non-uniform brightness?" The percentage of positive answers was taken as a measure of visibility. The visibility varied according to the parameters of the deviation and the gradient. With proper processing, a typical example of the plots of visibility versus the deviation and gradient was depicted in Fig. 3). In Fig. 3), L and L/ 2, denoted by , on the x-axis and $\mathrm{y}$ axis are the deviation and gradient respectively. The z-axis shows the percentage values for visibility.

Experimental results indicated that visibility was affected mainly by the spatial gradient of non-uniform brightness, rather than by the amount of deviation itself, that is, the amount of the difference in brightness. Other experiments resulted in similar plots. Thus, the spatial gradient in the non-uniform brightness distribution will be used to detect the defects in the proposed method based on human vision, which will be explained in next section.

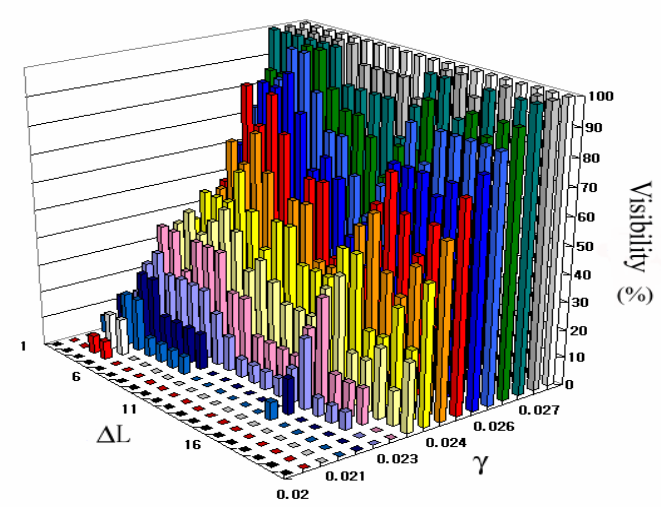

Fig. 3) Plot of visibility vs. deviation and gradient

\section{Detection of the Defects due to Non-uniform Brightness}

In this section, we explain a new method to detect the defects due to non-uniform brightness, which used the result that the visibility depends mainly on the spatial gradient of non-uniform brightness, rather than on the amount of brightness deviation. Thus, our proposed method uses the spatial gradient of brightness distribution to detect the defects. The detection method comprises four parts: contour extraction, calculation of the spatial gradient, the decision of defects, and the display of detected defects.

First, contours are extracted to obtain the information on the spatial distribution of nonuniform brightness. Although the term "contour" usually means the set of the pixels that have the same pixel value. ${ }^{[10]}$, here it refers to the collection of boundary pixels between adjoining pixel values. Thus, the more narrow the contours are, the steeper the spatial gradient of variation is. Hence, we can use the spatial gap between adjoining contours to obtain the spatial gradient of brightness. Practically, due to noise, the extracted contours should be smooth to avoid the distortion in calculating the gradient. We applied the morphological filter with OPEN and CLOSE 
to the captured images to obtain smooth contours. ${ }^{[11][12]}$

Since the spatial variation of non-uniform brightness is very gradual, it is unnecessary to determine the information on spatial distribution at all the pixels in the image. Thus, the next step in our proposed method is to find the appropriate pixel points at which the information on nonuniformity should be calculated and displayed.

To find the appropriate points, we first choose candidate points along the horizontal and vertical directions separately for simplicity in two-dimensional distribution of contours. Specifically, we render virtually horizontal lines and vertical lines with equal intervals. Considering the horizontal direction, we find the intersection points of virtually horizontal lines and the contours, and tentatively select the center points of the segmented lines between the intersection points. Thus, the area where the brightness varies rapidly will be crowded with the selected points, whereas the points will be sparse or even absent in the area where there is little variation in brightness. For the crowded area, there would be no need to calculate the gradient for every point. Hence, we find a representative point, for each region segmented by the intersection points of horizontal and vertical lines, by choosing a new pixel point at the centroid of the tentatively selected points, if there is more than one selected point. Note that the number of virtually rendered lines should be an important factor in the performance of the detection algorithm. If the number of lines is too small, the defect may be missed, whereas having too many lines may be time-consuming. We find experimentally the proper number of lines with which almost all of the non-uniformity defects should be detected within a reasonable amount of computation time. For example, for the captured image of 640x480 pixels, 15 horizontal and 20 vertical lines seemed almost optimal.

Next, at each point selected in the previous step, the spatial gradient value is calculated using the reciprocal of the mean value of the distances, called gap lengths, between adjoining contours around the point. In the proposed method, we obtain the gap lengths between the adjoining contours for horizontal and vertical direction separately as the distances between the intersection points mentioned above. Combining the horizontal and vertical gradient values yields the spatial gradient vector. Finally, we find the existence of defects by deciding whether or not the magnitude of the gradient vector exceeds a specified threshold, for each selected point. When the magnitude of the vector is larger than a specified value for a point, then it is decided that a non-uniformity defect should exist in the region around the point. And the information on the defect is displayed from the magnitude and orientation of the gradient vector.

\section{Experimental Results and Analysis}

To check how effective the proposed method is in detecting defects in actual LCD panels, several experiments were conducted using sample LCD panels with non-uniformity defects. First, images of an LCD displaying a mid-tone frame image were captured by a CCD camera for LCD panels with non-uniformity defects, of which a typical example was already shown in Fig. 1). Fig. 4), shows the drawing resulting from the contour extraction method, where the contours were depicted together. However, when we used these contours to find the information on the non-uniform brightness, they were distorted due to the noise. After removing the noise effect by applying proper morphological filters to the captured images, smooth contours could be extracted as shown in Fig. 5). Also, the selected points for the information on nonuniformity brightness was found according to the method mentioned earlier, which is shown in Fig. 6). 


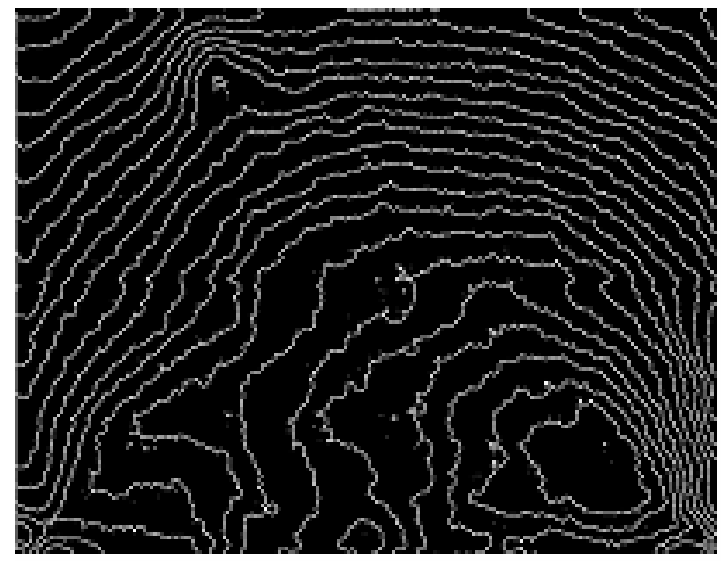

Fig. 4) Contours extracted from the captured image

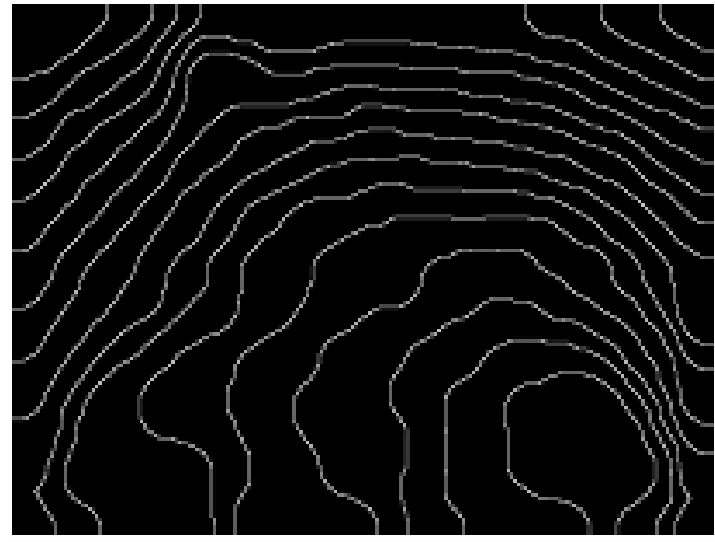

Fig. 5) Contours extracted from morphological filtered image

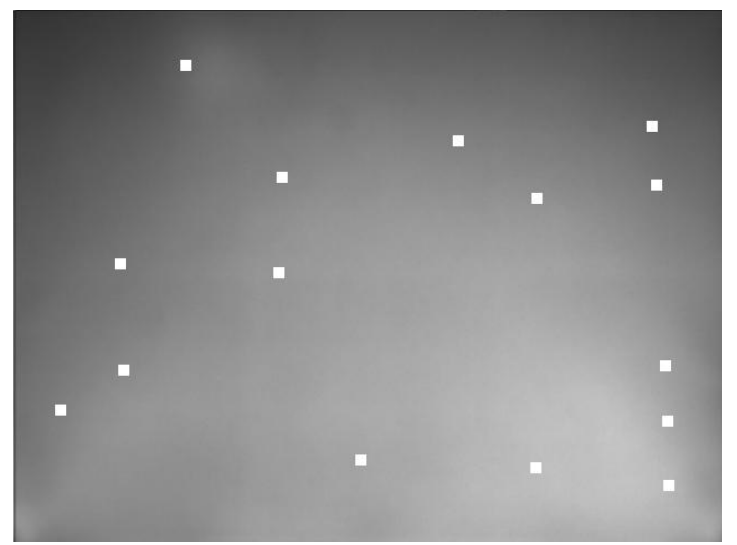

Fig. 6) Selected points for the display of nonuniformity information

The final result of the detection method is shown in Fig. 7), where the detected defects are displayed using arrows. The length of arrows indicates the magnitude of the gradient vector and the direction of the arrows is the direction of brightness variation. White dotted circles show easily the region of non-uniformity defects. Compared with raw image of Fig. 1), the nonuniformity defects that are detected by the proposed method seem to be consistent with the visible defects. The figure looks similar to atmosphere pressure plots for weather forecasting, where wind speed as well as direction can be estimated by a pressure gradient.

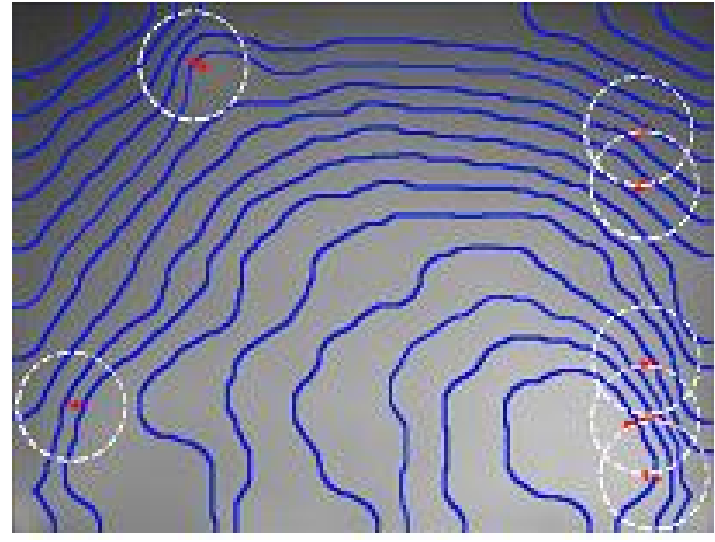

Fig. 7) Display of detected defects and nonuniform area

Via a series of experimental works for LCD panels with several types of non-uniformity defects, it can be seen that almost all the observable defects could be detected by the proposed method. Of course, the defects that are detected by the proposed method involve more than the non-uniformity defects that had been detected by human inspectors. The reason for this is that human inspectors tend to detect only a few major defects, since the existence of defects might be more important than the exact number of defects or more detailed information about the defects.

\section{Conclusion}

We proposed a method to detect defects due to spatially non-uniform brightness on LCD 
panels. The detection method is based on human vision so that some subjective assessment experiments were conducted to investigate the correlation between non-uniform brightness on a displayed image and how easily observable it is. Experiments revealed that visibility depends mainly on the spatial gradient of brightness variation. Thus, in the proposed method, the spatial gradient was used to detect defects due to non-uniform brightness. The detection method comprises four parts: contour extraction, spatial gradient calculation, decision of defects, and display of defects. Applying the method to the images captured from practical LCD panels produced results consistent with detection by a human inspector. Also, it was shown that the gradient values calculated in the proposed method can be possible criteria for defect decision.

The objective of the research is to develop a method of detecting defects due to nonuniformity in high quality display devices, based on the characteristics of the human visual system, and to apply the defect detection algorithm to automated detection systems via machine vision. With systematic analysis of the characteristics of human vision for visibility and of the categorization of blot shaped defects, a refined algorithm of spatial gradient in brightness variation in non-uniform displays will be developed for detection of defects due to nonuniformity, for both rendered and captured images.

\section{References}

1. Y. Mori, K. Tanahashi, R. Yoshitake-shi, S. Tsuji, "Extraction and Evaluation of Mura Images in Liquid Crystal Displays," Proceedings of SPIE, 4471, pp. 299-306, 2001

2. Fumihiko Saitoh, "A Method for Detecting Pixel Defects in LCD Visual Inspection System," Journal of the Japan Society for Precision Engineering, 63, No.4, pp. 520524, 1997
3. D. M. Regan, Human Perception of Objects, Sinauer Associates, 2000

4. Ulrich Eisemann, "Influence of Spatial Luminance Interactions on Perceived Brightness," Proceedings of SPIE, 4300, pp. 184-190, 2001

5. R. Jain, R. Kasturi, B. Schunck, Machine Vision, McGraw-Hill, 1995

6. Colin Martindale, Cognitive Psychology, Thomson Learning, 1994

7. Saitoh, F., "Uneven Area Defects Inspection on LCD Display Using Multiple Resolute Images," Journal of the Japan Society for Precision Engineering, 63, No.5, pp. 647651, 1997

8. Robson, J. G. \& Graham, N., "Probability Summation and Regional Variations in Contrast Sensitivity Curves across the Visual Field," Vision Research, 21, pp. 408-418, 1981

9. Wilbur B. Davenport Jr., Probability and Random Processes, McGraw-Hill, 1970

10. C. Gonzalez, R. E. Woods, Digital Image Processing, R. C. Gonzalez, R. E. Woods, Addison-Wesley, 1993

11. Arthur Weeks, Fundamental of Electronic Image Processing, SPIE/IEEE Series on Imaging Science \& Technology, 1998

12. Gregory A. Baxes, Digital Image Processing : Principles and applications, John Wiley \& Sons, 1994 